\title{
Influencia de agendas y estudio de framing en el marco electoral del Procés de Cataluña
}

\section{Influence of the agenda and framing study in the electoral framework of the Procés of Catalonia}

Concha Pérez-Curiel. Universidad de Sevilla. España.

cperez1@us.es

$[\mathrm{CV}]$ (1) $\mathrm{G}$

Gloria Jiménez-Marín. Universidad de Sevilla. España.

gloria_jimenez@us.es

$[\mathrm{CV}]$ (1) $\mathrm{G}$

Irene García Medina. Glasgow Caledonian University. Reino Unido.

irene.garcia2@gcu.ac.uk

$[\mathrm{CV}]$ (1) $\mathrm{G}$

Agradecimientos: Grupo de Investigación Communication and Social Sciences (SEJ-619). Universidad de Sevilla. Manuel Ignacio Lancharro Montiel, investigador y experto en Análisis Estadístico Digital.

Cómo citar este artículo / Referencia normalizada

Pérez-Curiel, C., Jiménez-Marín, G. y García Medina, I. (2020). Influencia de agendas y estudio de framing en el marco electoral del Procés de Cataluña. Revista Latina de Comunicación Social, 75, 27-51. https://www.doi.org/10.4185/RLCS-2020-1415

\section{RESUMEN}

Introducción: Momentos convulsos y de cambio definen el contexto político catalán. El tema de la independencia monopoliza la cobertura mediática de España y el Centro de Investigaciones Sociológicas (CIS) constata un aumento sin precedentes de la preocupación ciudadana (2017). Metodología: Se analiza la hibridación entre agendas (política, mediática y pública) a partir de una muestra específica de 526 tweets de candidatos independentistas y constitucionalistas, 107 portadas de prensa y 4 informes del CIS, con objeto de conocer la equivalencia de temas y los efectos en la población. Resultados: El asunto de Cataluña es el tema protagonista en Twitter, en las portadas de prensa y un $29 \%$ de los españoles, lo sitúa como primer problema en las encuestas. En paralelo, el frame de conflicto se sitúa como eje del discurso. Discusión y conclusiones: Se confirma la proyección de la agenda política digital en la agenda setting, así como la influencia en la decisión y prioridad de los asuntos de interés público, registrados en los sondeos poblacionales. Un efecto que se agudiza especialmente en situaciones de conflicto político, vinculadas al Proceso de Independencia de Cataluña.

PALABRAS CLAVE: Procés de Cataluña, influencers políticos; agenda setting; framing; Twitter; CIS. 


\begin{abstract}
Introduction: Moments of convulsion and change define the Catalan political context. The issue of independence monopolises the media coverage of Spain and the Centre for Sociological Research (CIS) notes an unprecedented increase in citizen concern (2017). Methodology: The hybridisation between agendas (political, media and public) is analysed on the basis of a specific sample of 526 tweets of candidates for independence and constitutionalism, 107 press covers and 4 CIS reports, in order to ascertain the equivalence of issues and the effects on the population. Results: The issue of Catalonia is the main topic on Twitter, on the press covers and 29\% of Spaniards, places it as the first problem in the polls. At the same time, the frame of conflict is at the heart of the discourse. Discussion and conclusions: The projection of the digital political agenda in the agenda setting is confirmed, as well as the influence on the decision and priority of matters of public interest, recorded in population polls. This effect is particularly acute in situations of political conflict linked to Catalonia's Independence Process.
\end{abstract}

KEYWORDS: Procés of Catalonia; political influencers; agenda setting; framing; Twitter; CIS.

\title{
CONTENIDO
}

1. Introducción. 1.1. Políticos y Medios. Influencers de la agenda pública. 1.2. El conflicto como framing. Estrategia de los medios y efectos en la ciudadanía. 2. Método. 2.1. Variables de procedimiento. 3. Resultados. 3.1. Primera fase. 3.1.1. Bloque cuantitativo numérico. 3.1.2. Bloque cualitativo temático y de framing. 3.2. Segunda fase. 3.2.1. Equivalencias y efectos de la agenda mediática sobre la agenda pública. 3.3. Tercera fase. 4. Discusión y conclusiones. 5. Referencias bibliográficas.

Traducción de Paul Jeremy Turner (PhD, University of Kent, England, United Kingdom).

\section{Introducción}

El 6 de septiembre de 2017 se aprueba en el Parlament de Catalunya la Ley de Referéndum y marca el inicio de un nuevo conflicto. Estatuto, Declaración de Soberanía, Ley de Consulta, Sentencias del Tribunal Constitucional y Artículo 155 anteceden a la convocatoria de unas elecciones autonómicas (21D) forzadas por la confrontación Cataluña/Estado (Carrasco-Polaino; Villar-Cirujano; TejedorFuentes, 2018, Pérez-Curiel; García-Gordillo, 2018). En este contexto, la prensa nacional y catalana define una selección y encuadre (framing) de noticias sobre el proceso independentista y electoral que, según los datos del CIS (2017), aumenta el interés público y puede influir en la disrupción y fragmentación social. Previamente, y en el espacio digital de Twitter, los candidatos de los partidos más votados en las elecciones (21D) publican tweets con un enfoque y una temática similar a la que más tarde proyectan los medios en sus portadas. Se genera una relación de causalidad que marca el conflicto catalán como tema de relevancia en las agendas política, mediática y pública.

La percepción de un bombardeo informativo continuado en el tiempo sobre una cuestión única -el Proceso de Independencia de Cataluña- con una cobertura mediática sin precedentes y unos índices de crecimiento de la preocupación social, registrados por los barómetros del Centro de Investigaciones Científicas (CIS, 2017), constituyen el soporte de la investigación. La premisa de una posible correlación de fuerzas determinada por el efecto de los medios de comunicación sobre la opinion pública (Teoría de la agenda-setting) confirmada inicialmente desde el ámbito científico (McCombs y Evatt, 1995) y avalada por estudios más recientes (Anduiza \& Bosch, 2012, D’Adamo, García Beadoux \& Freidenberg, 2007) se sitúa como eje hipotético del análisis.

Estudios previos ponen de manifiesto que los partidos políticos y especialmente sus candidatos han sido motores de influencia también en las redes sociales (Pérez-Curiel \& García Gordillo, 2018) y 
que los temas publicados por los líderes y más virales en Twitter han ocupado las portadas de la prensa de referencia (Adamic \& Glance, 2005; Reese, 2007; Scott, 2005). Una triple correlación de agendas y enfoques (D’ Adamo, García Beadoux \& Freidenberg, 2007) que afecta a todos los actores del proceso (políticos, medios y ciudadanos).

Sobre una muestra temporal que abarca desde el 7 de septiembre (día posterior a la aprobación del Referendúm por el Parlamento catalán) hasta el 22 de diciembre de 2017 (día después de las elecciones convocadas por el Gobierno Central)) se analiza la información publicada (107 portadas) por tres medios generalistas, dos de ámbito nacional (El País y El Mundo) y uno de ámbito autonómico (La Vanguardia).

La investigación establece como objetivo principal conocer la influencia y transferencia de agendas (política, mediática y pública) en el marco del Proceso de Independencia de Cataluña. Vinculados a este objetivo aparecen otros focos específicos de interés para el estudio:

- Observar qué tipo de temas y encuadres (framing) han predominado en la cobertura informativa sobre el proceso de Independencia de Cataluña y su equivalencia con los asuntos que marcan las preocupaciones ciudadanas.

- Relacionar el grado de influencia de la agenda política digital (temas que los candidatos de los partidos más votados en las elecciones catalanas han publicado en Twitter), sobre la agenda de los medios y de los ciudadanos.

\subsection{Políticos y medios. Influencers de la agenda pública}

La política en las democracias se desarrolla en la interrelación entre ciudadanos y políticos, entre ciudadanos y organizaciones políticas o entre ciudadanos e instituciones. Esa interrelación se produce en nuestras sociedades a través de los medios de comunicación con una capacidad de influencia que afecta a la esfera de los poderes del Estado y de los partidos. Además, crean opinión, y contribuyen al moldeamiento de la opinión ciudadana (Uriarte, 2010; Sanmartín, 2007). La agenda pública, relacionada directamente con las preocupaciones ciudadanas, se ve sometida a un proceso de mediatización determinado por la agenda setting (Bouza, 2006) mediante una correlación de temas, que no siempre coincide con las prioridades y el interés público.

Informar, moldear y orientar a la opinión pública y controlar al poder político son funciones primarias e intrínsecas que definen a las estructuras mediáticas (Gurevitch \& Blumler, 1999; Norris, 2001) y los convierte, especialmente en escenarios electorales, en generadores de opinión y en influencers activos por delante de gobiernos y partidos. Según recogen estudios previos 'influir' se convierte en la segunda tarea a la que los periodistas otorgan mayor importancia después de informar. La élite periodística se siente con legitimidad para representar a la opinión pública y la identifica con sus opiniones (Uriarte, 2000).

Sin embargo, el rol de influencer aparece previamente ligado a la figura del líder político (Fernández, Hernández-Santaolalla \& Sanz-Marcos, 2018; Labrecque, Markos y Milne, 2011) y a un discurso polarizado y unidireccional (Graham et al. 2013) en connivencia con los nuevos públicos digitales (Castells, 2009; Sampedro, 2014). El uso de Twitter como herramienta de persuasión y propaganda en contextos políticos y situaciones de crisis (Mancera Rueda \& Helfrich, 2014) se convierte en estrategia de autopromoción de sus propuestas y de distribución de sus propios contenidos, aplicando una lógica propagandística y basada en la viralización (Pérez-Curiel \& Limón Naharro, 2019; Dader \& Campos-Domínguez, 2017; Campos-Domínguez \& Calvo, 2017; López-García, 2016). 
Los candidatos más influyentes son aquellos capaces de crear una sinergia entre los canales de medios tradicionales y las redes sociales. Surge pues la agenda paralela de los medios, que reproduce el formato digital y refleja una equivalencia de temas y framing y con la agenda política. La hibridación de la esfera pública (Jenkins, 2008; Chadwick, 2013; Benkler, 2015) viene determinada por grupos hegemónicos y contrahegemónicos que luchan por visibilizarse, utilizando diferentes procesos de mediatización (Hjarvard, 2013; Holton et al., 2014) de los usuarios (Scolari, 2008).

El argumento del poder de los medios tradicionales para imponer la agenda, dada la confianza y credibilidad que generan en los ciudadanos, se intensifica en contextos políticos y electorales. Se refuerza la teoría intermedia de la agenda setting (Atwater \& Fico 1986; Reese, 1989) que analiza la relación entre agendas, la de los medios y la determinada por grupos de interés y/o movimientos sociales emergentes (Castells, 2012; Lazares, 1996) que luchan por transformar realidades "políticas, sociales y económicas" (Bermúdez \& Gallego, 2011). Son muy efectivos a la hora de transferir al público la importancia que tiene cada tema con la prioridad que consideran oportuna (Anduiza $\&$ Bosch, 2009).

En el caso concreto de Cataluña, inmersa en unas elecciones (21D) por efecto de la suma de conflictos ligados al Procés, se constata la influencia y el control de las instituciones y organizaciones en fijar la agenda, el proceso comportamental de los medios y el proceso participativo y social (Sánchez-Medero, 2016; Vliegenthart \& Walgrave, 2008). Los asuntos que marcan la agenda pública se derivan de los registros demoscópicos (CIS) correspondientes a cuestiones como ¿Cuál es, a su juicio, el principal problema que existe actualmente en Cataluña? ¿Y el segundo? ¿Y el tercero? La comparativa entre agendas muestra una correlación de rangos equivalentes con los temas que previamente los periódicos han publicado en sus portadas. La estructura y el contenido de las noticias tienen un importante efecto tanto sobre las cuestiones que los individuos consideran relevantes como sobre la complejidad con la que razonan sobre el tema (D'Adamo, García Beadoux \& Freidenberg, 2007). Por tanto, la respuesta ciudadana sobre las principales preocupaciones sociales en un contexto y un periodo temporal concreto pueden venir condicionadas por la influencia de los medios. Los asuntos de interés público, relacionados con los problemas que afectan directamente al bienestar de los ciudadanos (vivienda, empleo, educación, sanidad, corrupción política, pensiones, seguridad...) no cuentan con una presencia directamente proporcional a la que los medios atribuyen a situaciones de conflicto político y electoral.

Se plantea una primera pregunta de investigación:

PI1-¿Qué grado de influencia tienen los medios de comunicación sobre los ciudadanos en un contexto de conflicto político-electoral?

La hipótesis derivada (H1) manifiesta que la población catalana y española sitúan como asuntos de preocupación social aquellos temas previamente publicados por los medios de comunicación.

A esta perspectiva hay que añadir la influencia previa de la agenda política. Los medios le importan a la política y viceversa. Es algo inevitable. Están involucrados en el proceso de toma de decisiones e intervienen de distintas maneras, por ejemplo, dirigiendo la atención hacia determinados dominios públicos y ocultando otros (Soroka, Lawlor, Farnsworth \& Young, 2012), definiendo el mapa político que el público atenderá como verdadero y que, como tal, puede tener consecuencias en determinadas decisiones políticas (Cohen, 1993). Sin embargo, la revolución digital ha multiplicado en redes, especialmente en Twitter, el grado de influencia de los políticos, no solo sobre las comunidades de fans sino sobre la configuración de la agenda mediática (Montoya \& Vandehey, 2009; Pérez-Ortega, 2014; Rampersad, 2009). Se suma por tanto, la agenda inversa de los medios 
sociales (Aruguete, 2017; Wallsten, 2007; Meraz, 2011; Sung-Tae \& Young-hwan; 2007, CaseroRipollés; 2015).

Los líderes aprovechan los recursos digitales y el efecto multiplicador e instantáneo del mensaje en pro del interés electoral (López-García, 2016; Dader \& Campos-Domínguez, 2017; CamposDomínguez \& Calvo, 2017); los usuarios activan con likes, retweets y comentarios un discurso unidireccional del candidato frente a la bidireccionalidad que se le supone a la red y los medios tradicionales trasladan a portada la agenda política que mayor efecto ha provocado en los públicos.

Surge una segunda pregunta de investigación:

PI2. ¿Se nutren los medios tradicionales de los temas más difundidos por los políticos en Twitter?

En relación con la pregunta se formula la siguiente hipótesis (H2):

En el marco electoral del Procés, la tendencia de los medios es reproducir los temas-fuerza de los líderes políticos en Twitter y proyectar su influencia sobre la opinión pública.

\subsection{El conflicto como framing. Estrategia de los medios y efectos en la ciudadanía}

La última de las encrucijadas políticas a las que se ha enfrentado España, con una implicación real en su futura organización como Estado, ha sido el denominado Pro-cés en Cataluña, con la jornada considerada clave por sus promotores del referéndum del 1 de octubre de 2017, en el que se preguntaba a los ciudadanos catalanes si estaban a favor de la independencia y la "desconexión" de España (Carrasco-Polaino, Villar-Cirujano \& Tejedor-Fuentes, 2018). El proceso de independencia ha cobrado si cabe más fuerza en un contexto de mediatización y digitalización nunca antes contemplado. La cadena de actuaciones del Gobierno de España con la aplicación del artículo 155 de la Constitución, la disolución de las Cortes y la convocatoria de las elecciones del 21 de diciembre dibujan un escenario incierto. El informe del Centre d'Estudis d'Opinió computaba en 2017 un $40,2 \%$ de encuestados que consideraban que Cataluña debería ser un Estado independiente, un 27,4\% una Comunidad autónoma, un $21,9 \%$ un Estado federal y un 4,6\% una región $(C E O, 2017)$. La comparativa con estudios anteriores $(C E O, 2016)$ constata un afianzamiento de las posiciones independentistas e incluso de otros modelos territoriales por delante del modelo constitucional establecido. La reforma del Estatut (mayo de 2003) marca el inicio del denominado Procés. Aprobada, tras Referendum (junio de 2006) por el 73\% de los catalanes, defiende una mayor autonomía política de la comunidad, así como el reconocimiento de una posición singular de Cataluña dentro del Estado español.

El punto máximo de confrontación se sitúa en el contexto electoral anticipado por el Gobierno Central, que enfrenta y divide a partidos y ciudadanos. Un conflicto ideológico-político y una repercusión social que desde las redes sociales y medios de comunicación se ha proyectado fuera de Cataluña y que ha sobrepasado también las fronteras de España.

El establecimiento de la agenda, la selección de los issues-noticia y la influencia en receptores se refuerzan de forma complementaria con la teoría del framing y la interpretación de los marcos. Qué se cuenta viene determinado por Cómo se cuenta, un efecto framing que puede explicar el protagonismo de los medios en la opinión pública. A pesar del vasto campo de estudios sobre framing (Marín Zamora, 2014; Oller, 2014; López-Rabadán \& Vicente-Mariño, 2013; Sádaba, 2008; Weaver, 2007) es escasa la investigación en contextos políticos sobre cómo se produce el 'establecimiento de los encuadres' (frame setting). El estudio del encuadre permite indagar en el ejercicio de la influencia política (Entman, 2007; Iyengar, 2005), en la práctica del poder (Reese, 
2001) y de las dinámicas comunicativas dentro de una campaña política (Valera, 2014; Rodríguez \& Castromil, 2010).

Se produce una equivalencia de encuadres en la agenda y el conflicto se erige como primer tema de preocupación ciudadana, por delante de factores económicos, religiosos o de interés humano (Sábada \& Rodríguez, 2007). Los datos estadísticos de opinión poblacional publicados por el CIS (informes de septiembre-diciembre 2017) señalan como prioridad el conflicto político vinculado al Procés, que alcanza su máximo exponente durante el mes de octubre (referéndum ilegal, la comparecencia de Felipe VI, la fuga de empresas, la aprobación del artículo 155 y la convocatoria de elecciones catalanas). Un encuadre que también ha marcado la información política y mediática. En todos los casos, el factor cultural y social que rodea a los individuos ocupa una posición a tener en cuenta en la categorización e interpretación de las opiniones. Los medios de comunicación parecen lo suficientemente influyentes como para atraer la atención de los individuos y los públicos, pero esta influencia está moderada por la predisposición de los individuos, sus esquemas de interpretación de la realidad y otras características de su entorno social (Kinder, 2007).

Aplicado a la investigación, conceptos como nacionalismo, independentismo o segregación frente a otros como unidad, constitución y estado configuran una parte de la historia y la identidad transcultural de Cataluña; sin embargo, el devenir de acontecimientos políticos provocados por el Procés, en los que la participación activa de los sujetos se considera un valor añadido para los partidos constitucionalistas e independentistas, ha dibujado un encuadre mediático directamente relacionado con la división y la confrontación de las partes. La teoría del framing permite a los medios diseñar estrategias que incrementen el poder persuasivo inherente a la comunicación política e influir en el comportamiento del público, principalmente en el voto (Scheufele \& Tewksbury, 2007).

El establecimiento de la agenda y la configuración de los marcos durante periodos electorales (McCombs, 2005; Rodríguez \& Bouza, 2007) responde a enfoques mediáticos enfrentados; la prensa seleccionada para el estudio enfatiza aquellos issues que representan su posición ideológica y crea un discurso dirigido a una audiencia copartícipe. El Procés, ocupa desde el primer momento y de forma unánime las principales cabeceras informativas de España (El País y El Mundo) y de Cataluña (La Vanguardia), así como las portadas de los principales medios extranjeros, como un acontecimiento de notable relevancia histórica (Carrasco-Polaino, Villar-Cirujano \& Tejedor-Fuentes, 2018). Un encuadre único, el conflicto de la independencia, del que se deriva una temática relacionada con los códigos más habituales en las portadas (Gobierno Central, Generalitat, independentismo, constitucionalismo, aplicación del 155, situación de Puigdemont, políticos encarcelados, actuación judicial, salida de empresas de Cataluña, partidos y resultados electorales) describe la radiografía de agenda y framing del proceso.

En torno a la teoría de framing, se genera una tercera pregunta de investigación:

PI3. ¿Influye en la opinión ciudadana el frame de conflicto político que protagoniza las noticias relacionadas con la independencia de Cataluña?

Una tercera hipótesis (H3) plantea el conflicto político como encuadre principal utilizado por los periodistas en el proceso de selección e interpretación de las noticias

\section{Método}

A partir de una metodología triangulada de análisis de contenido cuantitativo y cualitativo (Wimmer \& Dominik, 1996; Krippendorff, 2004; Flick, 2004; Callejo, 2010), de enfoque comparado (Hallin \& 
Mancini, 2004), se diseña un muestreo que abarca desde el 7 de septiembre de 2017, fecha posterior a la aprobación de la Ley de Referendum por el Parlament de Cataluña hasta el 22 de diciembre, día después de las elecciones autonómicas convocadas por el Gobierno Central, puntos álgidos de conflicto político y social.

El método responde a las siguientes fases:

1. Se analizan las noticias que presentan la variable "Independencia de Cataluña", publicadas en portada (107) por los periódicos nacionales y autonómicos (El País, El Mundo y La Vanguardia) más leídos en España durante 2017 (OJD). El proceso se complementa con un estudio de framing para investigar los encuadres de selección e interpretación de los medios.

2. Se registran las equivalencias de la agenda mediática con la agenda pública. Se accede a los informes del Centro de Investigaciones Científicas correspondientes al último cuatrimestre de 2017 (septiembre-diciembre) que coincide con los episodios de mayor movilización política de Cataluña, previos a la convocatoria electoral (21D). Se seleccionan los tres primeros asuntos que los ciudadanos españoles han marcado como ejes de preocupación social y se analiza la correlación con lo publicado en las portadas de prensa.

3. Se relacionan los resultados obtenidos en las dos fases previas con los temas publicados en Twitter por los candidatos de los partidos más votados durante las elecciones catalanas (21D) en los periódicos seleccionados. Sobre una muestra específica de 526 tweets, relacionados con la marca Independencia de Cataluña se localizan las posibles equivalencias temáticas y de encuadre. Las cuentas personales corresponden a los candidatos de perfil constitucionalista:@InesArrimadas,de Ciudadanos; @miqueliceta,del PSC; y@Albiol_XG, del PP; y de perfil independentista: @KRLS, de Junts XCat; @martarovira, de ERC; y @ XavierDomenech, de Comú-Podems.

Los datos se extraen a través de la aplicación Twitonomy.

El programa elegido para el procesamiento de datos y la creación de tablas y gráficas de contingencia, de frecuencia y cruzadas, es el IBM SPSS Statistics, Versión 26. La fiabilidad de los intercodificadores se ha calculado con la fórmula Pi de Scott, alcanzando un nivel de 0,98.

\subsection{Variables de procedimiento}

Análisis cuantitativo numérico: Refleja el cómputo sobre las noticias publicadas en las portadas de los medios, con referencia a la Independencia de Cataluña.

Tabla 1. Codificación del análisis de contenido cuantitativo.

\begin{tabular}{|l|c|c|c|}
\hline Referencia en portada & Noticia principal & Noticia secundaria & Espacio en portada \\
\hline & 01 & 02 & 03 \\
\hline
\end{tabular}

Fuente: elaboración propia.

Análisis cualitativo de temas y encuadres: Refleja los issues principales sujetos al código Independencia de Cataluña aparecidos en las portadas de la prensa más leída, en la cuenta de Twitter de los candidatos con más representación (número de votos) y en los barómetros del CIS, correspondientes al último cuatrimestre de 2017. 
Tabla 2. Codificación por temas.

\begin{tabular}{|c|c|c|c|c|c|}
\hline Bloques & $\begin{array}{c}\text { Independencia/ } \\
\text { Constitución }\end{array}$ & $\begin{array}{c}\text { Gobierno de } \\
\text { España/ } \\
\text { Cataluña }\end{array}$ & $\begin{array}{c}\text { Aplicación } \\
\mathbf{1 5 5}\end{array}$ & $\begin{array}{c}\text { Huida de } \\
\text { Puigdemont/ } \\
\text { Políticos presos }\end{array}$ & $\begin{array}{c}\text { Salida de } \\
\text { empresas de } \\
\text { Cataluña }\end{array}$ \\
\hline Portadas & & & & & \\
\hline CIS & & & & & \\
\hline Twitter & & & & & \\
\hline
\end{tabular}

Fuente: elaboración propia.

Estos temas se clasifican en función de los frames susceptibles de aparecer en cualquier noticia (Neuman, Just \& Cringler, 1992; Iyengar \& Simon 1993), vinculados a un proceso de selección, énfasis, exclusión y elaboración del periodista.

Tabla 3. Codificación por frames.

\begin{tabular}{|c|l|l|l|l|l|}
\hline & De conflicto & $\begin{array}{c}\text { De interés } \\
\text { humano }\end{array}$ & $\begin{array}{c}\text { De consecuencias } \\
\text { económicas }\end{array}$ & De juicio moral & $\begin{array}{c}\text { De atribución de } \\
\text { responsabilidad }\end{array}$ \\
\hline Portadas & & & & & \\
\hline CIS & & & & & \\
\hline Twitter & & & & & \\
\hline
\end{tabular}

Fuente: elaboración propia.

La correlación triple de agendas (política/mediática/pública) y la transferencia de temas que afectan a la opinión ciudadana determina dónde radican las preocupaciones sociales en situaciones de conflicto político, describe la radiografía de Cataluña durante esta etapa del Procés y refuerza la teoría de la influencia de los centros de poder. La coincidencia con procesos electorales aumenta la posibilidad de disfunción de la agenda pública determinada por la acción de políticos y medios.

\section{Resultados}

El proceso de comprobación de niveles de influencia y correlación de agendas se estructura en tres fases, cada una de las cuales integra bloques de cuantificación (numérico) y/o de cualificación (temático y de encuadre). Los datos corresponden a la franja septiembre-diciembre de 2017, vinculada a un conjunto de conflictos políticos relacionados con el Proceso de Independencia de Cataluña.

\subsection{Primera fase}

\subsubsection{Bloque cuantitativo numérico}

El análisis estadístico mide el número de noticias publicadas en la portada de los tres periódicos, su clasificación como noticia principal o secundaria, así como el espacio que ocupan, en orden a la importancia que el medio otorga al acontecimiento. 


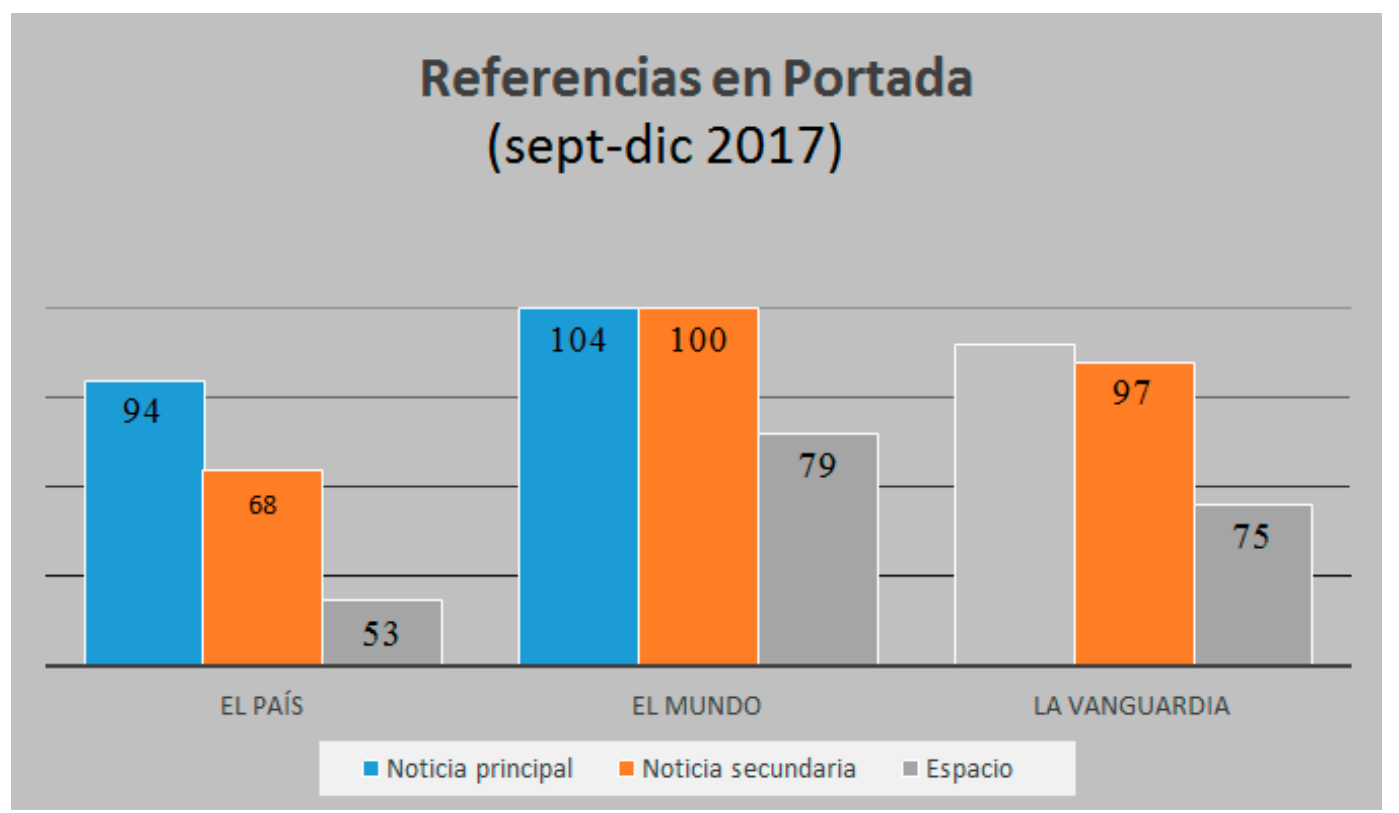

Gráfico 1: variable ' $\mathrm{N}^{\mathrm{o}}$ de noticias' en portada.

Fuente: elaboración propia.

El Mundo es el medio que realiza la mayor cobertura del tema Independencia de Cataluña, apareciendo como noticia principal y secundaria (104/100) respecto a la totalidad de portadas analizadas (107). Le sigue La Vanguardia (100/97) y, en tercer lugar, El País, con una cobertura menor (94/68) que no deja de ser representativa. El espacio que ocupa la información publicada es también directamente proporcional en los tres casos. De este modo, El Mundo (79\%), seguido por $L a$ Vanguardia (75\%) y El País (53\%), dedican una presencia destacada del tema de Cataluña.

De todos los meses de la muestra, octubre concentra el mayor cúmulo de conflictos relacionados con el Procés y publicados como noticia principal en portada (32/107). En los meses previos y posteriores, la cobertura disminuye, especialmente en diciembre, un mes monopolizado por la campaña electoral. Frente a ello, las noticias secundarias continúan captando el interés de la portada.

\subsubsection{Bloque cualitativo temático y de framing}

El contexto político del Procés describe núcleos temáticos temporales. En el mes de septiembre el hecho noticioso de referencia es la aprobación de la Ley de Referéndum en el Parlament catalán; octubre destaca por la celebración del Referéndum, la comparecencia del rey de Felipe VI, la aplicación del artículo 155 y la convocatoria de elecciones catalanas; en noviembre se producen sucesos múltiples como la encarcelación de miembros del gobierno catalán, el exilio europeo de Puigdemont, la precampaña electoral, la sospecha de la injerencia rusa en el proceso soberanista, y la caída del comercio interior en Cataluña; y cierra el mes de diciembre con las elecciones anticipadas (21D), hecho que redujo el interés por otros asuntos del proceso catalanista.

La comparativa de las portadas mediáticas constata por un lado, la equivalencia de temas y por otro, la coincidencia de enfoques y marcos de interpretación (framing), independientemente del perfil ideológico del medio. Los periódicos sitúan el caso de la Independencia de Cataluña como issue principal del que se derivan otros específicos. 
RLCS, Revista Latina de Comunicación Social, 75, 27-51

[Investigación] DOI: 10.4185/RLCS-2020-1415| ISSN 1138-5820 | Año 2020

Tabla 4. Tipología de temas y cobertura en portada (\% septiembre-diciembre).

\begin{tabular}{|c|c|c|c|c|c|c|}
\hline Bloques & $\begin{array}{c}\text { Independencia/ } \\
\text { Estado }\end{array}$ & $\begin{array}{c}\text { Gobierno de } \\
\text { España/ } \\
\text { Cataluña }\end{array}$ & Aplicación 155 & $\begin{array}{c}\text { Huida de } \\
\text { Puigdemont/ } \\
\text { Políticos presos }\end{array}$ & $\begin{array}{c}\text { Salida } \\
\text { empresas de } \\
\text { Cataluña }\end{array}$ \\
\hline El Mundo & $12,3 \%$ & $17,1 \%$ & $25,3 \%$ & $26,7 \%$ & $10,2 \%$ & $8,4 \%$ \\
\hline El País & $13,0 \%$ & $18,3 \%$ & $21,6 \%$ & $22,0 \%$ & $15,4 \%$ & $9,8 \%$ \\
\hline La Vanguardia & $12,2 \%$ & $15,0 \%$ & $22,4 \%$ & $29,5 \%$ & $14,2 \%$ & $6,7 \%$ \\
\hline
\end{tabular}

Fuente: elaboración propia.

Todos los medios coinciden en señalar las variables Aplicación del 155 y Huida de Puigdemont/ Políticos Presos como focos centrales de sus ediciones. Son consideradas como líneas de confrontación de gobiernos y partidos y la prensa traslada a las portadas una polarización del asunto, en función de su posición ideológica, con efectos en los electores.

La Vanguardia destaca el bloque Huida de Puigdemont/Políticos presos como noticia estrella (29,5\%), seguido por El Mundo (26,7\%) y El País (22,5\%); se consideran, de este modo, los dos temas que mayor grado de división y opinión generaron entre políticos, medios y ciudadanos, y una consecuencia directa de la aplicación del artículo 155, segundo tema-fuerza de portada. Esta disposición refuerza la autoridad del Gobierno de España y anula la legitimidad de la Generalitat, máxima institución política en Cataluña. La Vanguardia (22,4\%), como prensa autonómica, lo trata desde la perspectiva de conflicto abierto entre fuerzas independentistas y constitucionalistas. El enfoque de El Mundo (25,3\%) y El País $(21,6 \%)$ subraya la necesidad de intervención del Gobierno Central ante el cariz de los acontecimientos.

El bloque relativo a Salida de Empresas de Cataluña cuenta con mayor cobertura por parte de los tres periódicos, especialmente de El País (15,4\%) y La Vanguardia (14,2\%) que lo analizan como conflicto económico causado tras la actuación del Gobierno independentista. Es reseñable el valor adjudicado a las elecciones, un issue que suele concentrar la atención de los medios en otros contextos pero que en el marco de conflicto de la independencia de Cataluña pierde protagonismo en favor del resto de ítems. Los bloques (Independencia/Constitución y Gobierno de España/Cataluña) presentan un tratamiento equilibrado en todas las portadas y además son marca compartida de la información sobre el Procés.

Ideología y Framing constituyen un tándem que condiciona el modo de informar de cada medio. El estudio de caso de Cataluña revela la importancia de un tema analizado desde la perspectiva de conflicto. Por delante del espacio y el número de noticias publicadas por la prensa, se sitúa el modo en que los periodistas informan de los hechos. Qué aspectos se tratan y cómo (énfasis), cuáles se obvian (exclusión), qué fuentes se seleccionan y cómo se explican e interpretan los hechos en orden al discurso establecido. 


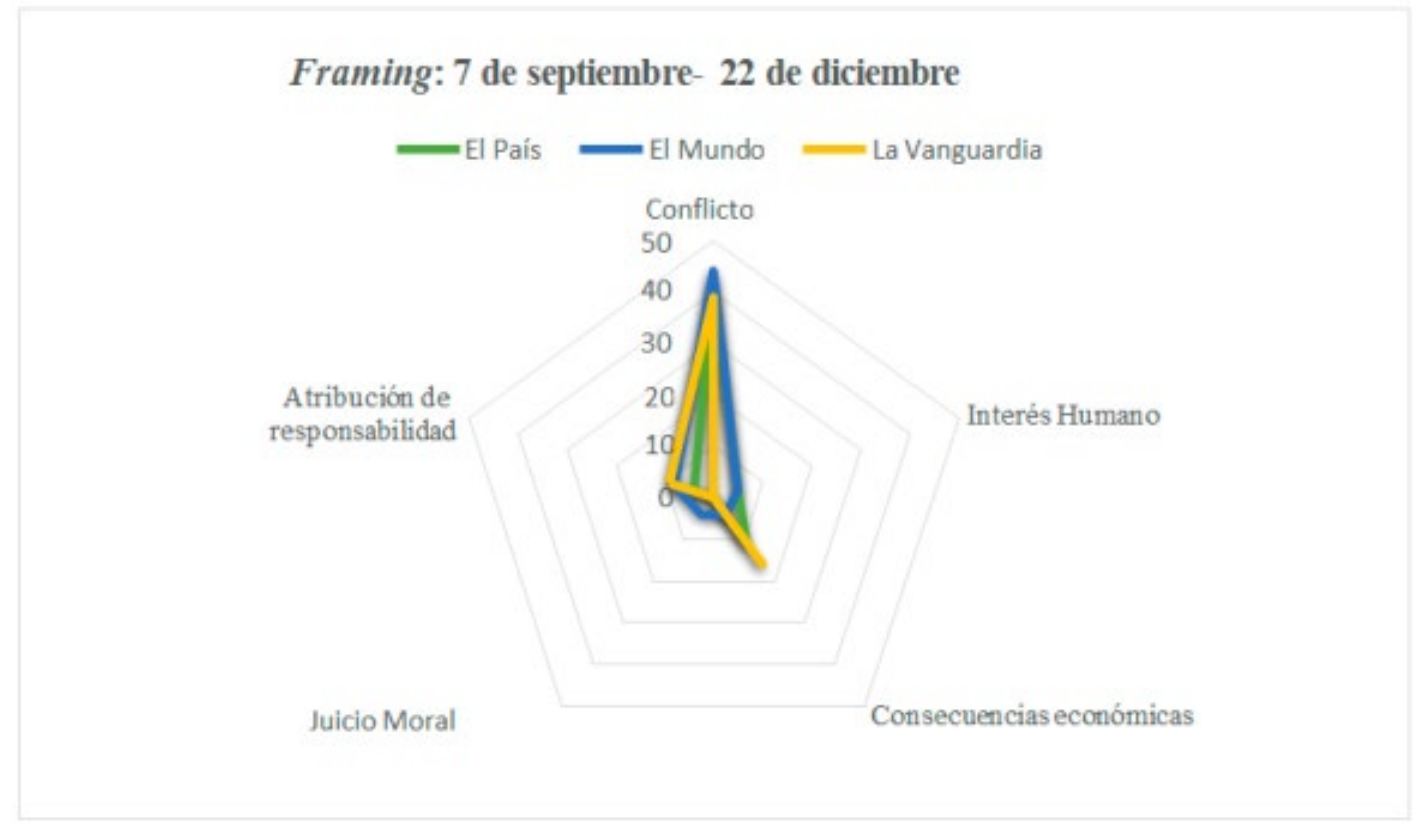

Gráfico 2: tipología de framing y cobertura en portada.

Fuente: elaboración propia.

Como señala la gráfica, el conflicto es el encuadre más utilizado por la prensa, por delante del resto de frames. La confrontación política entre partidarios de la independencia y defensores del Estado es una constante informativa. Los asuntos que alcanzan mayor énfasis son los relacionados con el Referéndum del 1-O, la comparecencia del Rey, el traslado de las sedes de múltiples empresas, la puesta en marcha del proceso judicial, la aplicación del artículo 155 y la convocatoria de elecciones. Los tres periódicos priorizan el frame de conflicto frente al resto, alcanzando los picos más altos, las variables Aplicación del 155, Huida de Puigdemont y Encarcelamiento de políticos. Destaca el frame de atribución de responsabilidad que los dos primeros concentran en el bloque a favor de la independencia mientras que El País enfatiza el frame de consecuencias económicas, relacionado con la salida de empresas de Cataluña.

Los tres medios consideran el frame de interés humano en la selección y elaboración de la información de portada. Destaca El Mundo con noticias sobre la división de familias por ideología o el adoctrinamiento de alumnos en las escuelas catalanas. 


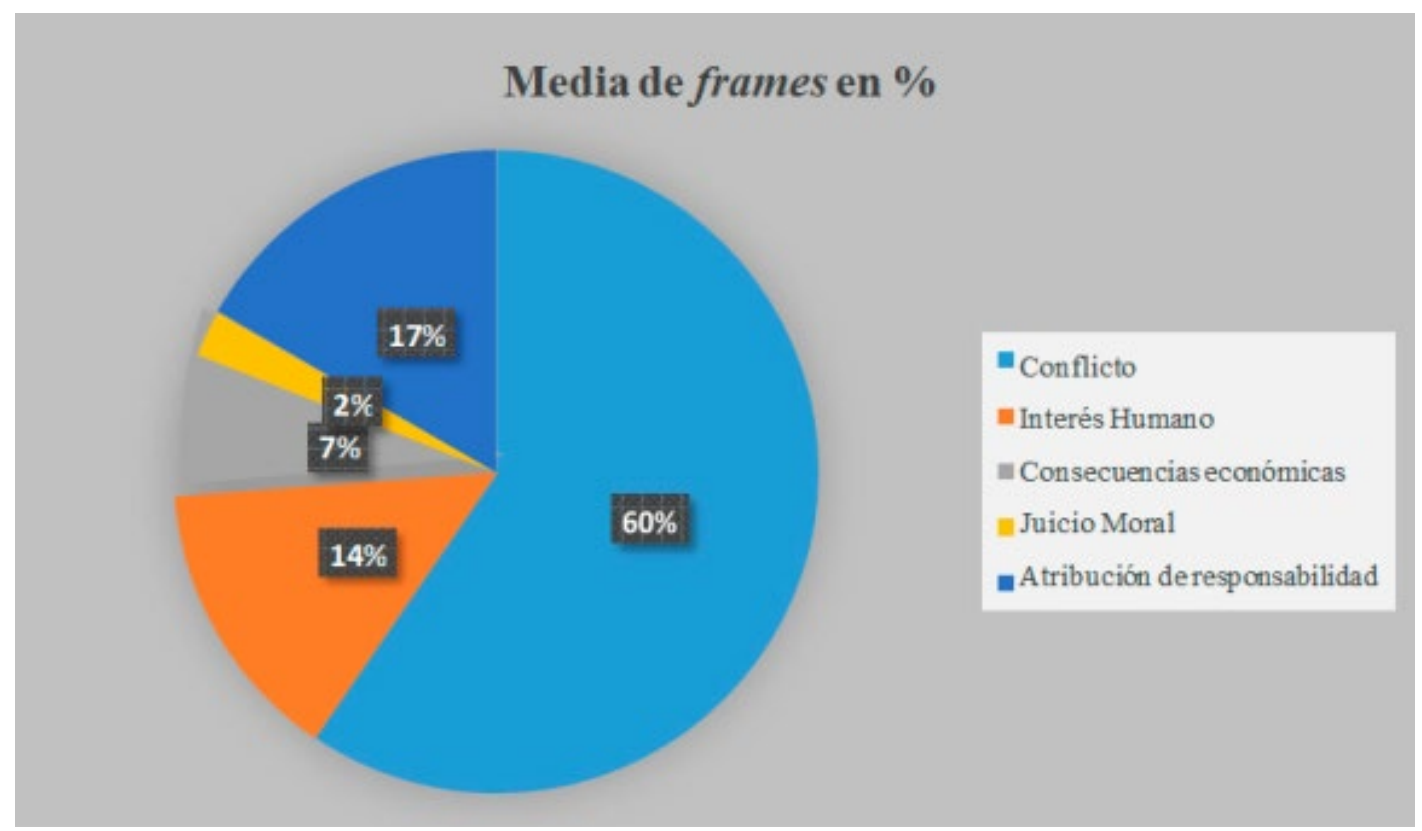

Gráfico 3: media y \% de frames en portada.

Fuente: elaboración propia.

Un resumen de la media de encuadres durante el periodo investigado refleja el protagonismo del frame de conflicto. (60\%) respecto al resto. En orden de importancia le siguen la atribución de responsabilidad (15\%), una variable focalizada en la actuación del gobierno, de los partidos y de las asociaciones ciudadanas de perfil independentista, el frame de interés humano (12\%), equivalente a la fragmentación social que el enfrentamiento político ha provocado en la población y las consecuencias económicas (11\%) derivadas de la decisión de las empresas de abandonar Cataluña, dada la situación de inestabilidad derivada del conflicto. El frame de juicio moral, basado en la posición deontológica del medio ante la actitud de las partes implicadas, alcanza un porcentaje mínimo $(2 \%)$ que podría converger con la variable de atribución de responsabilidad.

\subsection{Segunda fase}

\subsubsection{Equivalencias y efectos de la agenda mediática sobre la agenda pública}

Con el objetivo de conocer la influencia del encuadre y de los temas publicados por la prensa en la opinión pública, se analizan los informes del Centro de Investigaciones científicas correspondientes a los meses de muestreo (septiembre-diciembre de 2017). Los resultados de la encuesta definen qué es lo que más preocupa en primer, segundo y tercer lugar, así como la media resultante. 


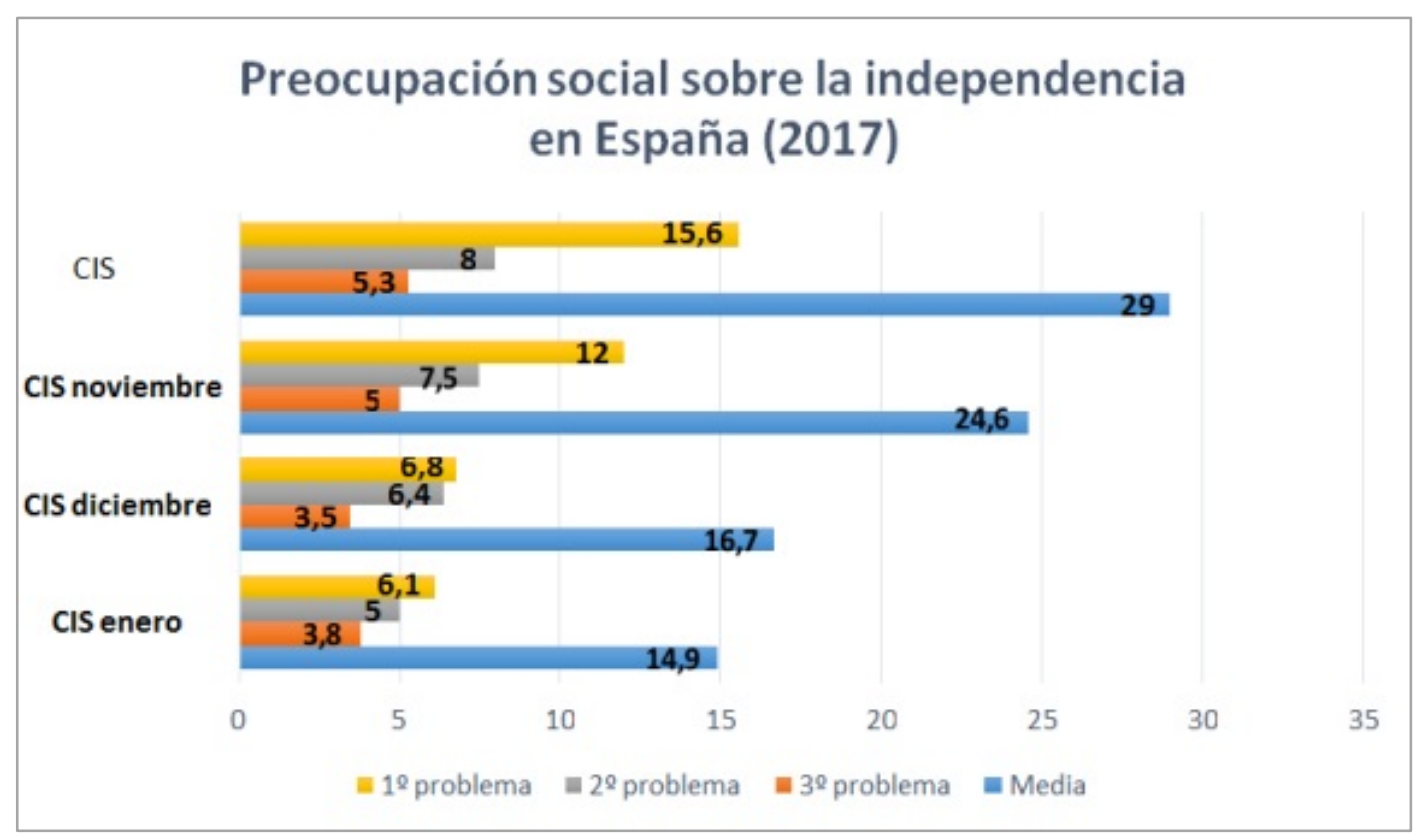

Gráfico 4: niveles de preocupación social ante la independencia en España.

Fuente: elaboración propia.

Como se puede observar en la encuesta de octubre, el 29\% de los españoles señalan la independencia de Cataluña como una de las tres primeras preocupaciones de la población. El desglose lo clasifica como primer problema $(15,6 \%)$, como segundo $(8 \%)$ y como tercero $(5,3 \%)$. La recogida de datos (2-11 de octubre) coincide con la aprobación de la Ley de Referéndum, las impresiones de la jornada del 1 de octubre, o la comparecencia del Rey. Por ofrecer una mayor perspectiva, en el CIS del mes anterior (septiembre), la independencia de Cataluña ocupa el octavo problema del escalafón (7,8\%) por lo que se produce un incremento notable en cuanto a nivel de preocupación.

En el CIS de noviembre, el $24,5 \%$ de los españoles consideran la independencia de Cataluña como asunto de interés, situándolo como primer problema (12\%), como segundo $(7,5 \%)$ y como tercero $(5 \%)$, respectivamente. A pesar de la aprobación del 155, la convocatoria de elecciones, o la renuncia de las empresas a permanecer en Cataluña se constata un descenso (de segundo a cuarto puesto) como foco de interés respecto al mes anterior.

Durante los meses de diciembre $(16,7 \%)$ y de enero $(14,9 \%)$ se produce una bajada significativa del interés ciudadano ante el tema de la independencia; en ambos casos, la celebración de elecciones, se considera un punto de inflexión que relativiza la actitud de la población ante el asunto. La horquilla de porcentajes de ambos meses lo señala como primer problema $(6,8 \% / 6,1 \%)$, como segundo $(6,4 \% / 5 \%)$ y como tercero $(3,5 \% / 3,8 \%)$, ocupando el quinto puesto en el ranking de problemas prioritarios para los españoles.

En línea con el estudio, se propone analizar la opinión de la población catalana. Se rescatan los datos del CIS relativos a comunidades autónomas y se diseña una tabla de variables cruzadas (preocupación ciudadana/independencia de Cataluña). 


\section{Preocupación social sobre la independencia en Cataluña (2017)}

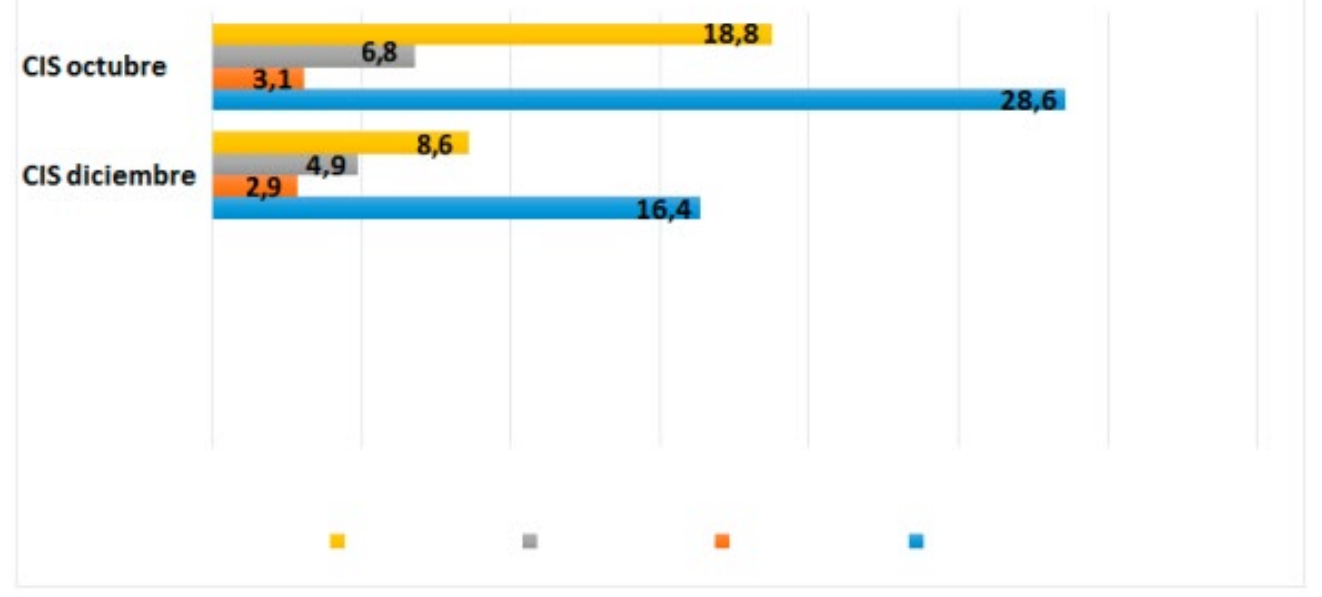

Gráfico 5: niveles de preocupación social ante la independencia en Cataluña.

Fuente: elaboración propia.

Los datos por comunidades autónomas, dentro del periodo de análisis, solo fueron ofrecidos en los barómetros de octubre y diciembre, con lo que los resultados muestran más claramente la tendencia descendente.

En el CIS de octubre, el tema de la independencia se situó como el tercer problema $(28,6 \%)$. Es decir, la preocupación social es ligeramente inferior a la media estatal. No obstante, el porcentaje de catalanes que lo señalaron como el primer problema social fue del $18,8 \%$, tres puntos más que la media estatal. Los guarismos bajan en la designación como segundo $(6,8 \%)$ y tercer problema $(3,1 \%)$ alrededor de dos puntos por debajo de la media nacional. En el CIS de diciembre se observa una evolución descendente importante, ya que la preocupación por la independencia se coloca como el quinto problema de los catalanes $(16,4 \%)$, con una valoración similar a la media estatal.

\subsection{Tercera fase}

Investigaciones previas ponen de relieve la influencia del discurso de los políticos sobre los usuarios en Twitter (Pérez-Curiel \& García-Gordillo, 2018), así como los efectos sobre los medios de comunicación (Pérez-Curiel \& Limón-Naharro, 2019). La agenda de los medios puede estar a su vez previamente determinada por la agenda política.

Con el fin de conocer si la prensa reproduce en portada los temas publicados por los políticos, se han seleccionado un total de 526 tweets vinculados a la marca Independencia de Cataluña, publicados en Twitter por los candidatos de perfil constitucionalista (@InesArrimadas,@miqueliceta y @Albiol_XG) y de perfil independentista (@KRLS,@martarovira y @XavierDomenech), de los partidos más votados durante las elecciones catalanas y con un mayor índice de seguiminento de usuarios en la red. 
RLCS, Revista Latina de Comunicación Social, 75, 27-51

[Investigación] DOI: 10.4185/RLCS-2020-1415| ISSN 1138-5820| Año 2020

Tabla 5. Número de tweets, seguidores y métricas (a fecha de muestreo).

\begin{tabular}{|c|c|c|c|c|c|c|}
\hline Candidatos & $\begin{array}{l}\text { Puigdemont } \\
\text { JuntsxCat }\end{array}$ & $\begin{array}{c}\text { Arrimadas } \\
\text { C's }\end{array}$ & $\begin{array}{c}\text { Rovira } \\
\text { ERC }\end{array}$ & $\begin{array}{l}\text { Iceta } \\
\text { PSC }\end{array}$ & $\begin{array}{c}\text { Albiol } \\
\text { PP }\end{array}$ & $\begin{array}{c}\text { Domènech } \\
\text { En Comú- } \\
\text { Podem }\end{array}$ \\
\hline Seguidores & 670.056 & 401.319 & 164.232 & 81.210 & 63.160 & 62.345 \\
\hline Tweets & 88 & 66 & 52 & 174 & 75 & 71 \\
\hline Me Gusta & 68.800 & 7.300 & 6.139 & 227 & 2914 & 3416 \\
\hline Retweets & 34.000 & 3.282 & 2405 & 216 & 1988 & 2117 \\
\hline Comentarios & 4.317 & 1.549 & 1235 & 79 & 2855 & 1865 \\
\hline
\end{tabular}

Fuente: elaboración propia.

Los datos muestran la gran diferencia de tweets publicados por el candidato Miquel Iceta del PSC (174) frente al resto de líderes. En esta línea, conviene comentar que la mayoría corresponde a tweets no propios, apoyados en links, vídeos, imágenes y otros recursos, que Iceta difunde desde su cuenta personal.

Salvando esta excepción, es Carl Puigdemont, líder independentista de Junts per Cat y presidente de la Generalitat hasta la convocatoria de elecciones, el candidato que publica mayor número de tweets relacionados con la marca Independencia de Cataluña (88) y que alcanza un mayor número de seguidores y de métricas (Me gusta, RT y Comentarios). Puigdemont se considera además el líder que mejor uso realiza de las herramientas que proporciona Twitter (hashtags, menciones, enlaces, memes, recursos audiovisuales...) para reforzar el mensaje.

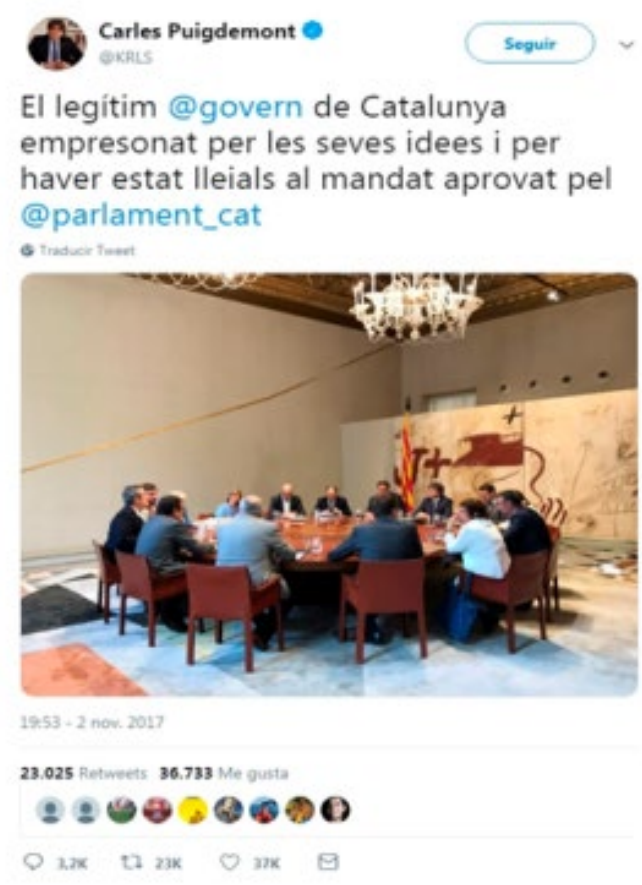

Figura 1: Tweet políticos presos.

Fuente: Twitter. 
RLCS, Revista Latina de Comunicación Social, 75, 27-51

[Investigación] DOI: 10.4185/RLCS-2020-1415| ISSN 1138-5820 | Año 2020

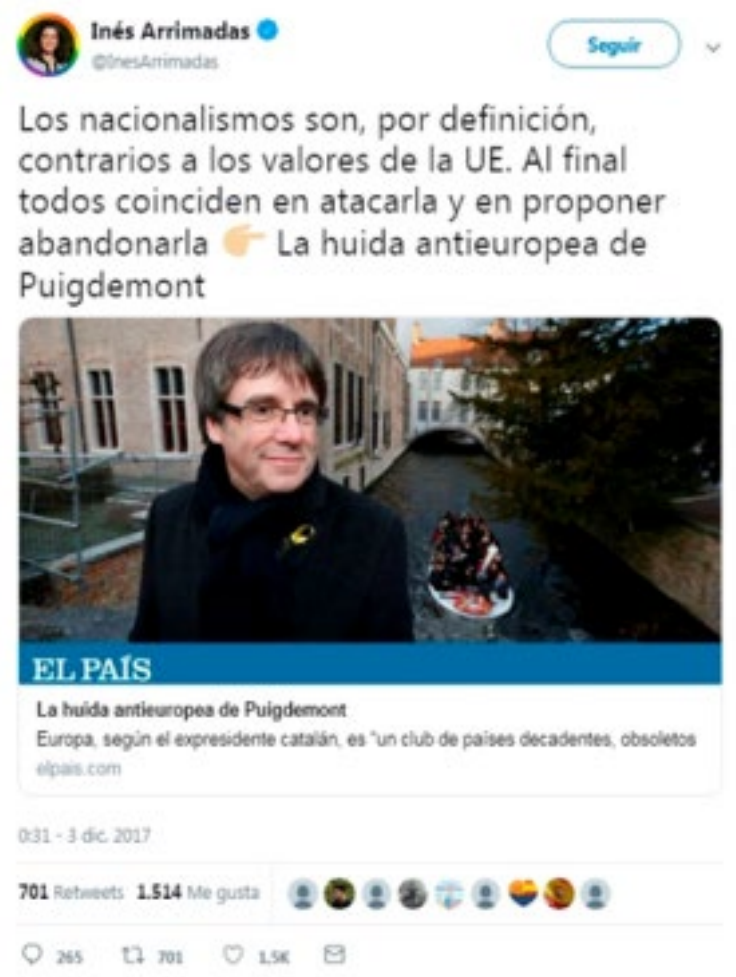

Figura 2: Tweet huida de Puigdemont.

Fuente: Twitter.

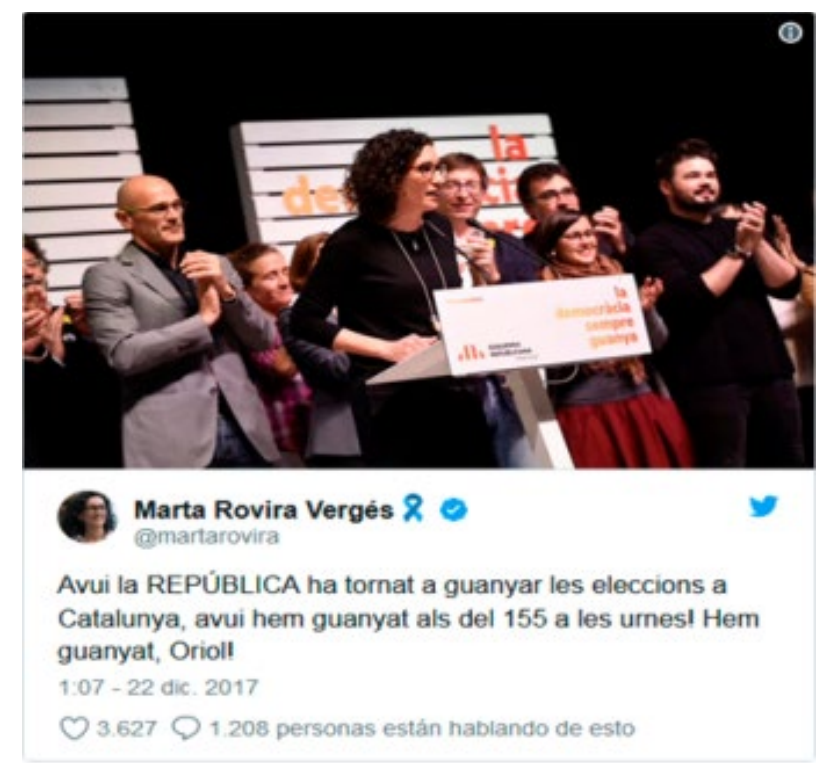

Figura 3: Tweet Artículo 155.

Fuente: Twitter. 


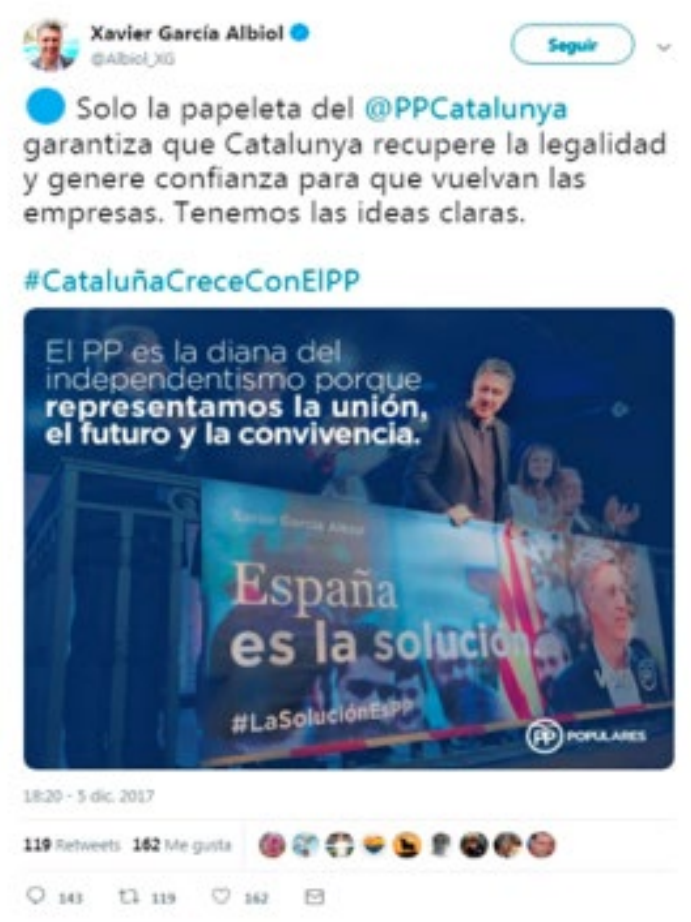

Figura 4: Tweet salida de empresas.

Fuente: Twitter.

En general, los tweets publicados por los perfiles seleccionados se centran en contenidos equivalentes a los que más tarde publican los periódicos que, a su vez, se reflejan en las encuestas del CIS, donde el tema de Cataluña se sitúa como una de las principales preocupaciones ciudadanas en los meses de muestreo.

La tabla refleja sobre el total de la muestra seleccionada, el porcentaje destinados a los temas más difundidos desde las cuentas personales de los candidatos y el porcentaje de presencia en las portadas de prensa. El cálculo se estima sobre el 100\% de los ítems específicos relacionados con la independencia.

Tabla 6. Media en \% de temas tratados en Twitter y prensa.

\begin{tabular}{|l|c|c|c|}
\hline \multicolumn{1}{|c|}{ Temática } & Twitter & Prensa & Valores Medios \\
\hline Independencia/Estado & 10,9 & 12,1 & 11,5 \\
\hline Gobierno de España & 12,3 & 14,0 & 13,1 \\
\hline Gobierno de Cataluña & 9,4 & 8,2 & 8,8 \\
\hline Artículo 155 & 19,1 & 17,2 & 18,1 \\
\hline Huida de Puigdemont & 17,3 & 15,7 & 16,5 \\
\hline Políticos Presos & 13,0 & 10,4 & 11,7 \\
\hline Salida de empresas & 6,1 & 8,5 & 7,3 \\
\hline Elecciones & 8,0 & 10,0 & 9 \\
\hline Otros & 3,7 & 4,2 & 3,9 \\
\hline
\end{tabular}

Fuente: elaboración propia.

El artículo $155(18,1 \%)$ y la huida de Puigdemont (16,5\%) alcanzan la media más alta. Le siguen temas como Gobierno de España (13,1\%), Independencia/Estado (11,5\%) y Políticos presos (11,7\%). 
Elecciones (9\%), Salida de empresas $(7,3 \%)$ y Otros $(3,9 \%)$ ocupan un tercer bloque en cuanto al interés de políticos y medios.

Los porcentajes indican una doble equivalencia. Por un lado, se observa la similitud de los temas publicados y por otro la coincidencia en cuanto a la prioridad otorgada a los asuntos relacionados con la independencia en Cataluña. Es una característica común a todos los contenidos difundidos en Twitter y en la prensa que, posteriormente reflejan los barómetros del CIS.

Tabla 7. Media en \% problemas sociales recogidos por el CIS.

\begin{tabular}{|l|c|c|c|c|c|}
\hline $\begin{array}{c}\text { Independencia de } \\
\text { Cataluña }\end{array}$ & $\begin{array}{c}\text { Primer } \\
\text { Problema }\end{array}$ & $\begin{array}{c}\text { Segundo } \\
\text { Problema }\end{array}$ & $\begin{array}{c}\text { Tercer } \\
\text { Problema }\end{array}$ & Total & $\begin{array}{c}\text { Valores } \\
\text { Medios }\end{array}$ \\
\hline Octubre & 15,6 & 8 & 5,3 & 28,9 & 9,6 \\
\hline Noviembre & 12 & 7,5 & 5 & 24,5 & 8,1 \\
\hline Diciembre & 6,8 & 6,4 & 3,5 & 16,7 & 5,5 \\
\hline Enero & 6,1 & 5 & 3,8 & 14,9 & 4,9 \\
\hline
\end{tabular}

Fuente: elaboración propia.

Aunque el paro aparece como primer problema señalado por los ciudadanos en todos los meses de muestreo, el conflicto catalán ocupa el segundo tema de interés durante el mes de octubre (28,9\%), el cuarto lugar en el CIS de noviembre $(24,5 \%)$ y experimenta una bajada con respecto a los meses de diciembre $(16,7 \%)$ y enero $(14,9 \%)$, situándose en quinto lugar entre los asuntos de interés público. En todos los casos supera la posición del mes de septiembre donde aparece como octavo problema (7,8\%).

El cálculo de la media hace referencia a la combinación porcentual de los datos ofrecidos por los barómetros correspondientes al periodo octubre-enero de 2017 y la consideración del tema como primer, segundo o tercer problema. Se constata que el tema de la Independencia de Cataluña, recogido por el CIS durante la franja septiembre-octubre, registra un incremento como problema poblacional, con picos más elevados en los meses de mayor confrontación Cataluña/Estado, que va disminuyendo conforme avanza el proceso. Las publicaciones en Twitter y en prensa sobre temas relacionados con la independencia de Cataluña siguen la misma fluctuación de tiempos que los asuntos publicados por el CIS. Una equivalencia directamente proporcional que puede justificar la influencia y transferencia de agendas.

\section{Discusión y conclusiones}

En el marco del conflicto que rodea al Procés de Cataluña (septiembre-diciembre de 2017) y desde una perspectiva global, los resultados confirman la influencia y correlación de agendas (política/mediática/pública). Los ciudadanos, a través de la encuestación del Centro de Investigaciones Sociológicas, exponen como asuntos prioritarios aquellos que previamente han publicado los medios de comunicación. De la misma forma, la prensa seleccionada para el estudio (El Mundo, El País y La Vanguardia) lleva a portada los principales temas que los políticos han difundido en Twitter durante el periodo de muestra establecido.

Como respuesta al objetivo general, el estudio confirma la alineación temática y de framing que se inicia en la red, marca la agenda setting y provoca efectos en los ciudadanos. El incremento de la preocupación de la población española y catalana recogido por el CIS puede responder entre otros factores a una situación de conflicto político (Procés) y electoral (21D) sumada al criterio de selección de temas de los candidatos políticos en Twitter y a la agenda setting marcada por los medios. 
Las conclusiones se estructuran atendiendo a los objetivos específicos planteados:

1. El estudio confirma la influencia que los medios de comunicación analizados ejercen sobre los asuntos de máxima preocupación social registrados por el CIS.

Tal y como arrojan los resultados extraídos de los análisis cuantitativos sobre portadas y editoriales de El País, El Mundo y La Vanguardia entre septiembre y diciembre de 2017, la cobertura mediática ha sido constante. Durante esos meses el asunto catalán monopolizó, prácticamente, todas las portadas y editoriales de los medios seleccionados, lo que ilustra que la agenda-setting estuvo muy condicionada por el asunto de la independencia de Cataluña.

Además, se constata la influencia y correlación entre agendas (mediática y pública), situándose el issue general (la independencia) y los temas específicos desglosados (Independencia/Estado, Gobierno de España, Gobierno de Cataluña, Artículo 155, Huida de Puigdemont, Políticos presos, Salida de Empresas y Elecciones) como preocupaciones sociales prioritarias en los barómetros del CIS, con resultados sin precedentes durante los meses de muestreo.

2. El análisis cualitativo del framing, derivado del seguimiento y medición de las 107 portadas seleccionadas, concluye que el encuadre que ha predominado es el de conflicto y, que este marco es el que destaca en los tres periódicos.

El conflicto, frente a otros encuadres (interés humano, consecuencias económicas, juicio moral, atribución de responsabilidad) es el vector que ha alineado las informaciones relativas al Procés, destacando fundamentalmente en la primera etapa de la investigación (septiembreoctubre). Este periodo refleja el mayor grado de preocupación social (CIS) y refuerza la hipótesis (H1) sobre la influencia de los medios de comunicación en la inquietud de los ciudadanos, en momentos de convulsión política y electoral.

Se produce una relación directamente proporcional entre el grado de conflicto y el nivel de problemática social considerado por la población. Si además, se suma el contexto de unas elecciones autonómicas en Cataluña (21D) convocadas por el Gobierno de España ante la crisis sobrevenida, aumenta en proporción aritmética el interés de los medios para seleccionar estos temas y decidir un encuadre, con efectos comprobados en los ciudadanos.

3. La temática de los mensajes publicados en Twitter por los candidatos de los partidos políticos más votados durante las elecciones catalanas (21D) coincide con los issues principales de las portadas de prensa y con los temas de mayor interés público, señalados por la ciudadanía.

El principio de causalidad, inicialmente vinculado a las redes, explica un recorrido de la influencia que sigue un esquema circular. Lo que se informa y difunde en Twitter, se traslada a los medios, provoca una reacción y una opinión en los públicos y a su vez puede tener también repercusión en la red.

El análisis de las cuentas personales de los políticos refleja una selección de temas relacionados directamente con el framing de conflicto, que dadas las herramientas que proporciona Twitter, se hacen virales y producen efectos en medios y audiencias.

Finalmente, se confirma el proceso de hibridación y transferencia de agendas (Aruguete, 2017; Chadwick, 2013); existe una correlación triangulada: los temas más recurridos por los líderes son a su vez los más difundidos por los periódicos y que alcanzan mayor interés público. Las portadas de prensa, en consonancia con Twitter, focalizan su atención en acontecimientos vinculados directamente con la Independencia de Cataluña (155, huida de Puigdemont, políticos encarcelados, salida de empresas de Cataluña), por delante de temas inherentes a los procesos electorales (programa, resultados o agenda política, entre otros).

En línea con futuras investigaciones se propone indagar en la teoría de la agenda inversa propulsada por los medios sociales, que activa un mecanismo de abajo a arriba, protagonizado por los ciudadanos y la sociedad civil, que puede condicionar la agenda mediática gracias al impacto y 
alcance del mensaje en las redes. De esta forma emergen oportunidades para el cambio social y político (Aruguete, 2017; Casero, 2015; Wallsten, 2007; Meraz, 2011; Sung-Tae \& Young-hwan, 2007).

Estudiar el activismo ciudadano on y off (Soengas \& Assif, 2017) y su influencia sobre políticos y medios (Howard \& Hussain, 2013) alterando los esquemas hasta ahora definidos, investigar el papel de los usuaios como prosumidores en las redes, o de las asociaciones ciudadanas (Corominas, 2017) como entes activos frente a los centros de poder, pueden ser también objeto de análisis.

Por último, avanzar en la definición de influencia e influencers (Pérez-Curiel \& García-Gordillo, 2018), en la localización de nuevas estrategias en Twitter (López-Meri, Marcos-García \& CaseroRipollés, 2017) y en el impacto del fact-checking sobre la agenda (Mazaira-Castro, Rúas-Araujo \& Puentes-Rivera, 2019; Wintersieck, 2017), conforman posibles líneas abiertas y de continuidad en el marco de la comunicación política.

\section{Referencias Bibliográficas}

Adamic, L. \& Glance, N. (2005). The political blogosphere and the 2004 presidential election. http://goo.gl/XhkSIO.

Anduiza, E. y Bosch, A. (2004). Comportamiento politico y electoral. Ariel Ciencia Política.

Aruguete, N. (2017). The agenda setting hypothesis in the new media environment. Comunicación $y$ Sociedad, 28, 35-58.

Atwater, T. \& Fico, F. (1986). Source reliance and use in reporting state government: A study of print and broadcast practices. Newspaper Research Journal, 8, 53-61.

Benkler, Y. (2015). La riqueza de las redes: cómo la producción social transforma los mercados y la libertad. Icaria.

Bermúdez, P. y Gallegos, A. (2011). Las teorías de la interacción social en los estudios sociológicos. http://goo.gl/bH7qI1

Bouza, F. (2006). La influencia de los medios de comunicación en la opinión pública. [Tesis Doctoral]. Universidad de La Coruña, España.

Carrasco-Polaino, R. Villar Cirujano, E. y Tejedor Fuentes, L. (2018). Twitter como herramienta de comunicación política en el contexto del referéndum independentista catalán: asociaciones ciudadanas frente a instituciones públicas. Icono 14, 16(1), 64-85.

https://doi.org/10.7195/ri14.v16il.1134

Campos-Domínguez, E. (2017). Twitter y la comunicación política. El profesional de la información, 26(5), 785-793. https://doi.org/10.3145/epi.2017.sep.01

Casero-Ripollés, A, (2015). Estrategias y prácticas comunicativas del activismo político en las redes sociales. Historia Y Comunicación Social, 20(2), 533-548.

Castells, M. (2012). Redes de indignación y esperanza: los movimientos sociales en la era de Internet. Alianza Editorial. 
Castells, M. (2009). Comunicación y poder. Alianza editorial.

Centre d'Estudis d'Opinió (2017). Baròmetre d'Opinió Política. $2 a$ ornada 2017 - REO 857. https://goo.gl/CJVNJs

Chadwick, A. (2013). The Hybrid Media System, Politics and Power. Oxford University Press.

Cohen, B. (1993). The Press and Foreign Policy. University of California.

Corominas, O. (2017). The struggle for the story in political discutes. The case of the $9 \mathrm{~N}$ participation process. El professional de la información, 26(5), 884-893.

D’Adamo, O., García Beaudoux, V. y Freidenberg, F. (2007). Medios de comunicación y opinión pública. McGraw-Hill.

Dader, J. L. y Campos-Domínguez, E. (2017). La búsqueda digital del voto. Cibercampañas electorales en España 2015-16. Tirant lo Blanch.

Entman, R.M. (2007). Framing Bias: Media in the Distribution of Power. Journal of Communication, 57, 163-173.

Fernández, J. D., Hernández-Santaolalla, V. y Sanz-Marcos, P. (2018). Influencers, marca personal e ideología política en Twitter. Cuadernos.info, 42, 19-37. https://doi.org/10.7764/cdi.42.1348

Flick, U. (2004). Introducción a la investigación cualitativa. Morata.

Gurevitch, M, \& Blumler, J. (1999). Political Communication Systems and democratic values, en J Lichtemberg (edit.). Democracy and the mass media. Cambridge University Press.

Hallin, C. \& Mancini, P. (2004). Comparing media systems. Three models of media and politics. Cambridge University Press.

Hjarvard, S. (2013). The mediatization of culture and society. Routledge.

Holton, A. E., Baek, K., Coddington, M. \& Yaschur, C. (2014). Seeking and sharing motivations for linking on Twitter. Communication Research Reports, 31(1), 33-40.

Howard, P.N. \& Hussain, M.M. (2013). Democracy's fourth wave? Digital media and the Arab Spring. Oxford University Press.

Iyengar, S. \& Simon, A. (1993). News coverage of the gulf crisis and public opinion: a study of agenda-setting, priming, and framing". Communication Research, 20, 365- 383.

Jenkins, H. (2008). Convergence Culture. La cultura de la convergencia en los medios de comunicación. Paidós.

Kinder, D. R, (2007). Curmudgeonly advice. Journal of Communication, 57, 155-162.

Krippendorff, K. (2004). Content analysis. Sage. 
Labrecque, L. Markos, E. \& Milne, G. (2011). Online personal branding: Processes, challenges, and implications. Journal of Interactive Marketing, 25(1), 37-50.

https://doi.org/10.1016/j.intmar.2010.09.002

Lazares, C. (1996). La teoría de las redes sociales. Papers, 48, 103-126.

López-García, G. (2016). 'New' vs 'old' leaderships: The campaign of Spanish general elections 2015 on Twitter. Comunicacion y sociedad, 29(3), 149-168.

https://doi.org/10.15581/003.29.3.149-168

López-Meri, A. Marcos-García, S. \& CaseroRipollés, A. (2017). What do politicians do on Twitter? Functions and communication strategies in the Spanish electoral campaing of 2016. El profesional de la información, 26(5), 795-804. https://doi.org/10.3145/epi.2017.sep.02

López-Rabadán, P. y Casero-Ripollés, A. (2012). La evolución de la agenda mediática española (1980-2010). Un análisis longitudinal de la portada de la prensa de referencia. Revista Latina de Comunicación Social, 67, 470-493.

Mancera Rueda, A. y Helfrich, U. (2014). La crisis en 140 caracteres: el discurso propagandístico en la red social Twitter. Cultura, Lenguaje y Representación, 12, 59-86.

https://doi.org/10.6035/CLR.2014.12.4

McCombs, M. y Evatt, D. (1995). Los temas y los aspectos: explorando una nueva dimensión de la Agenda-setting. Comunicación y Sociedad, 8(1), 7- 32.

McCombs, M. (2005). Look at Agenda Setting: past, present and future. Journalism Studies, 6(4), 543-557.

McQuail, D. (1992). La acción de los medios, los medios de comunicación y el interés público. Amorrortu.

Marín, J. A. y Zamora, R. (2014). Aproximación integradora en la investigación sobre la teoría del framing desde su condición multiparadigmática. Disertaciones: Anuario electrónico de estudios en Comunicación Social 7(1).

Mazaira-Castro, A., Rúas-Araújo, J. \& Puentes-Rivera, I. (2019). Fact-Checking en los debates electorales televisados de las elecciones generales de 2015 y 2016. Revista Latina de Comunicación Social, 74, 748 - 766. https://doi.org/10.4185/RLCS-2019-1355

Meraz, S. (2011). Using time series analysis to measure intermedia agenda-setting influence in traditional media and political blog networks. Journalism \& Mass Communication Quarterly, 88(1), 176-194. https://doi.org/10.1177/107769901108800110

Montoya, P. \& Vandehey, T. (2009). The Brand called you. Create a personal branding that wins attention and grows your business. McGraw-Hill.

Norris, P. (2001). Digital divide? Civic engagement, information poverty and the internet worldwide. Cambridge University Press. 
Oller, M. (2014). The triangle formed by framing, agenda-setting and metacoverage. Disertaciones: Anuario electrónico de estudios en Comunicación Social 7(1).

Pérez-Curiel, C. y García-Gordillo, M. (2018). Política de influencia y tendencia fake en Twitter. Efectos postelectorales (21D) en el marco del Procés en Cataluña. El profesional de la información, 27(5), 1030-1040.

Pérez-Curiel, C. y Limón Naharro, P. (2019). Influencers de la Política. Estudio de la marca personal de Donald Trump en Twitter y efectos en medios y usuarios. Communication \& Society, 32(1), 57-76.

Reese, S. D. (2007). The framing project: A bridging model for media research revisited. Journal of Communication, 57(1), 148-154. https://doi.org/10.1111/j.1460-2466.2006.00334.x

Reese, S. D. (1989). Inter-Media Influence and the Drug Issue: Converging on Cocaine. Communication Campaignsabout Drugs: Government, Media, and the Public, 5, 29-46.

Rodríguez, R. y Bouza, F. (2007). Cambios en las agendas y cambios en la Comunicación Política: encuadres (frames) y agendas. II Congreso de Comunicación Política. Universidad Complutense de Madrid.

Rodríguez, R. y Castromil, A.R. (2010). La circulación social de los encuadres periodísticos en tiempo de campaña electoral: Transmisión, influencia y atribución de responsabilidad. Revista de Estudios de Comunicación, ZER, 15(29), 193-212.

Sádaba, T. (2008). Framing: el encuadre de las noticias. El binomio terrorismo-medios. La Crujía.

Sádaba, T. y Rodríguez Virgili, J. (2007). La construcción de la agenda de los medios. El debate del estatut en la prensa española. Ámbitos. Revista Internacional de Comunicación, 16, 187-211.

Sampedro, V. (2014). El cuarto poder en red: por un periodismo (de código) libre. Icaria.

Sánchez-Medero, R. (2016). Comunicación politica: Nuevas dinámicas y ciudadanía permanente. Tecnos.

Sanmartín, A. (2007). El poder del cuarto poder: la influencia del marketing político en las democracias modernas. ECA: Estudios centroamericanos.

Scheufele, D. A. \& Tewksbury, D. (2007). Framing, Agenda Setting, and Priming: The Evolution of Three Media Effects Models. Journal of Communication, Vol. 57, pp. 9-20.

Scolari, C. (2008). Hipermediaciones. Elementos para una teoría de la comunicación digital interactiva. Gedisa.

Scott, D. T. (2005). Tempests of the blogosphere: Presidential campaign stories that failed to ignite mainstream media.

http://citeseerx.ist.psu.edu/viewdoc/download?doi=10.1.1.537.4209\&rep=rep1\&type=pdf.

Soengas, X. \& Assif, M. (2017). Cyberactivisim in the Process of Political and Social Change in Arab Countries. Comunicar, 53, 49-57. https://doi.org/10.3916/C53-2017-05 
Soroka, S., Lawlor, A., Farnsworth, S. \& Young, L. (2012). Mass Media and Policymaking, en E Araral, S Fritzen, M Howlett, M Ramesh y X Wu (Eds.). Routledge Handbook of Public Policy, 1-15. Routledge.

Sung-Tae, K. \& Young-hwan, L. (2007). New functions of Internet mediated agenda-setting: Korea. Journalism review, 1(2), 3-29.

Uriarte, E. (2010). Los medios de comunicación de masas y la opinión pública, en E. Uriarte (autor) y J. J. Linz (Col.), Introducción a la Ciencia Política (pp. 345-367). Tecnos.

Valera, L. (2014). Agenda building y frame promotion en la campaña electoral de 2011: La circulación del discurso entre partidos, medios y ciudadanos. [Doctoral dissertation]. University of Valencia, Spain. https://www.educacion.gob.es/teseo/imprimirFicheroTesis.do?fichero=51949.

Wallsten, K. (2007). Agenda setting and the blogos- phere: An analysis of the relationship between mainstream media and political blogs. Review of policy research, 24(6), 567-587. http://doi.org/10.1111/j.1541-1338.2007.00300.x

Weaver, D. H. (2007). Thoughts on agenda setting, framing, and priming. Journal of communication 57(1), 142-147.

Wimmer, D, y Dominick, J. R. (1996). La investigación científica de los medios de comunicación. Una introducción a sus métodos. Bosch.

Wintersieck, A. (2017). Impact of Fact-Checking During Electoral Debates. American Politics Research, 45(2), 304-331.

\section{AUTORES:}

\section{Concha Pérez Curiel}

Profesora Ayudante Doctora. Departamento de Periodismo II. Universidad de Sevilla. España.

Doctora por Universidad de Sevilla y profesora de Periodismo Político en Grado, dirige el Máster de Comunicación y Moda e imparte docencia en el Máster de Comunicación Institucional y Política y en el Máster de Estudios Europeos de la Universidad de Sevilla. Pertenece al grupo de investigación Communication \& Social Sciences (SEJ-619) y participa en el proyecto de investigación CSO201788620-P, financiado por el Ministerio de Ciencia, Innovación y Universidades del Gobierno de España y en el Proyecto "Ciberataques y soberanía: el nuevo desafío del orden internacional" (Programa Operativo FEDER Andalucía 2014-2020). Ha sido investigadora visitante en universidades internacionales de referencia como Central Saint Martins de Londres, Sacro Cuore y E-Campus (Milán) y de Porto (Portugal) y nacionales como la Universidad Complutense de Madrid o la Universidad del País Vasco. Trabaja sobre comunicación política, influencia y transferencia de agendas, nuevas narrativas digitales y efectos sobre los medios y los usuarios. Cuenta con publicaciones en revistas científicas como Communication and Society, El profesional de la información, Estudios sobre el Mensaje Periodístico y Prisma Social, entre otras.

cperez1@us.es

Índice H: 7

Orcid ID: https://orcid.org/0000-0002-1888-0451

Google Scholar: https://scholar.google.es/citations?hl=es\&user=wyp6bucAAAAJ 


\section{Gloria Jiménez Marín}

Profesora Titular de Universidad. Departamento de Comunicación Audiovisual y Publicidad. Universidad de Sevilla. España.

Doctora en Comunicación, Licenciada en Periodismo y Licenciada en Publicidad y RR.PP. por la Universidad de Sevilla, es Profesora Titular de Universidad en la Facultad de Comunicación de la misma universidad, además de colaboradora en la Universitat Oberta de Catalunya. Ha sido profesora profesora en la Universidad de Cádiz (SP), en la Universidad de Huelva (SP) y en la University of Wales (UK). Asimismo, ha sido profesora invitada en la University of California Berkeley (USA), en la Universitat Pompeu Fabra (SP) y en la Universidad Complutense de Madrid (SP). Es autora de varios libros sobre comunicación, autora de más de 40 comunicaciones a Congresos y de más de 35 artículos científicos, y directora de varias tesis doctorales, Es directora de la revista científica internacional IROCAMM - International Review Of Communication And Marketing Mix y ha sido, igualmente, presidenta, vocal y comité científico de varios congresos, simposios y jornadas científicas. Revisora de publicaciones científicas, a nivel internacional, de marketing y comunicación. Es miembro de La Academia de la Publicidad y directora del Grupo de Investigación SEJ-624 International Research Of Communication And Marketing Mix. Desde el punto de vista profesional ha trabajado en varios medios de comunicación (radio y prensa), en agencias de publicidad y en departamentos de comunicación de varias entidades hasta que irrumpió en su vida el mundo de la docencia e investigación universitaria.

gloria jimenez@us.es

\section{Indice $\mathrm{H}: 8$}

Orcid ID: http://orcid.org/0000-0003-0252-3975

Google Scholar: https://scholar.google.es/citations?user=pNLUjXUAAAAJ\&hl=es

\section{Irene García Medina}

Professor. Departamento de Business Management. Glasgow Caledonian University. Reino Unido.

Profesora de Marketing en la Glasgow Caledonian University (Glasgow, Reino Unido), Irene se licenció en Ciencias de la Información por la Universidad Complutense de Madrid, y es doctora en Marketing por la Universidad de Sophia Antipolis, (Francia) así como doctora en Relaciones Internacionales por la Universidad de Viena (Austria). En su labor como profesora, Irene ha impartido previamente clases a nivel de grado y postgrado en el área del marketing en la Universidad de Madeira (Portugal), en la Universidad de Vic y en la Universidad Pompeu Fabra de Barcelona. Respecto a su carrera profesional, ha trabajado, entre otros, como directora de Marketing en la empresa francesa VTDIM, como consultora para la Cámara de Comercio de Portugal o como jefa de Comunicación y Promoción de CORDIS (Comisión Europea). Irene ha impartido conferencias, seminarios y talleres en el área del marketing y la publicidad en varios países, además de publicar numerosos artículos y libros sobre esta materia. Sus principales intereses en el ámbito de la investigación se centran en el marketing móvil, el marketing digital, la comunicación digital, las redes sociales y el e-branding.

Irene.Garcia2@gcu.ac.uk

Índice $\mathrm{H}: 7$

Orcid ID: http://orcid.org/0000-0003-4424-3357

Google Scholar: https://scholar.google.es/citations?user=ZHazpv4AAAAJ\&hl=es\&oi=sra 